\title{
Effect of the exchange hole on the Gutzwiller approximation in one dimension
}

\author{
Balázs Hetényi, Hans Gerd Evertz, and Wolfgang von der Linden \\ Institut für Theoretische Physik, Technische Universität Graz, A-8010 Graz, Austria
}

\begin{abstract}
The Gutzwiller approximate solution to the Gutzwiller wavefunction yields exact results for the Gutzwiller wavefunction in the infinite dimensional limit. Implicit in the Gutzwiller approximation is an approximate local form of the fermion exchange hole. This approximate form is the same for all dimensions but is incorrect except in infinite dimensions. We implement the correct form for the exchange hole into the Gutzwiller approximation. We perform calculations on the one-dimensional Hubbard model at half-filling. They indicate that the implementation of the exchange hole already brings the Gutzwiller approximation into very close quantitative agreement with the results of the full Gutzwiller wavefunction. Metallicity as well as anti-ferromagnetism are recovered.

PACS numbers: 71.10.Fd,71.30+h,71.10.Ca
\end{abstract}

\section{INTRODUCTION}

The Hubbard model [1, 2, 3, 4] and its descendants have contributed greatly to our understanding of strongly correlated systems [5, 6, 7] and in particular of the metalinsulator transition 5] (MIT) exhibited by them. Early attempts [4, 8] to explain the MIT were based on the use of a projected wavefunction due to Gutzwiller (GWF). The GWF is a variational method whose starting point is a non-interacting wavefunction, in which double occupations and consequently charge fluctuations are suppressed.

Exact solutions to the GWF are known in one [9, 10] and infinite dimensions 10, 11, 12. In one dimension the exact solution of GWF is metallic, a conclusion which was shown [13] to be general for finite dimensions. The exact solution to the Hubbard model at half-filling in one-dimension [14] is insulating for all finite values of the interaction strength. Extended versions of the GWF with charge fluctuations can only account for insulating behavior when correlations between doubly occupied sites and empty sites are incorporated (bound excitons) [15, 16, 17].

The GWF is often treated via an approximation also due to Gutzwiller [4, 7, 8, 18] (GA). The GA predicts a MIT [8, 19] between a paramagnetic metal and a paramagnetic insulator (Brinkman-Rice transition) at half-filling, in contradiction with the exact Gutzwiller solution. The GA also does not account for antiferromagnetic correlations properly whereas exact diagonalizations have shown that the GWF, inspite of being based only on projecting out double occupations, reproduces anti-ferromagnetic correlations remarkably well [20]. On the other hand the GA corresponds to the exact solution of the GWF when the number of dimensions is infinite [10, 11, 12, 21]. An improved GA has previously been constructed by Metzner 22] where it is shown that self-energy corrections can restore metallicity. Another important study relevant here is that of van Dongen et al.[23] in which it is shown that metallicity can be recovered based on dimensional scaling arguments, however finite orders of perturbation theory are not sufficient to remove the Brinkman-Rice MIT. In two dimensions metallicity can be recovered [24, 25] via a diagrammatic summation method in which the error terms are estimated with high accuracy [26]. This has also been demonstrated numerically [27].

Interestingly, the exchange hole (pair-correlation function of particles with parallel spins, defined as $g\left(r_{i j}\right)=$ $\left\langle n_{i} n_{j}\right\rangle$ where the average is over the Fermi sea) for noninteracting electrons at half filling, applied in GA is independent of the physical dimension. It corresponds to the exact GWF result only in infinite dimensions (See Fig. 11). The crucial point here is that in finite dimensions the exchange hole extends over several lattice sites, while in GA it is restricted to the on-site term. The central motivation of the present paper is to show that the failure of GA is due to the over-simplified approximation of the exchange hole.

The GA consists of taking exchange into account in a combinatorial fashion. The configurations considered obey the Pauli principle in the sense that no two particles of the same spin can be found on the same site. On the other hand no other correlation effect exists between like spins in the GA. Hence the exchange hole is only local corresponding to the infinite dimensional case (Fig. 1). It is known, however (see for example Refs. 7] and 28]) that the exchange hole in a finite number of dimensions has a nontrivial functional form (Fig. 1).

The GA as well as extensions of it enjoy widespread use in a variety of strongly correlated problems. The Brinkman-Rice transition has been used by Vollhardt to describe the solid-liquid phase transition in $\mathrm{He}^{3}$ [19]. More recent applications of the GA include extension to the time-dependent case 29], implementation for the multi-band case [30, 31], ensembles with varying particle number (Bardeen-Cooper-Schrieffer wavefunction) [32], and the calculation of matrix elements between ground and excited states 33. Variants of the approximate solution have also been applied [34, 35, 36] in the resonating valence bond method [37, 38, 39], which is based on a completely projected Gutzwiller wavefunction.

In this paper we augment the GA with an improved treatment of the exchange hole. Our calculation samples the exact distribution in the occupation number rep- 


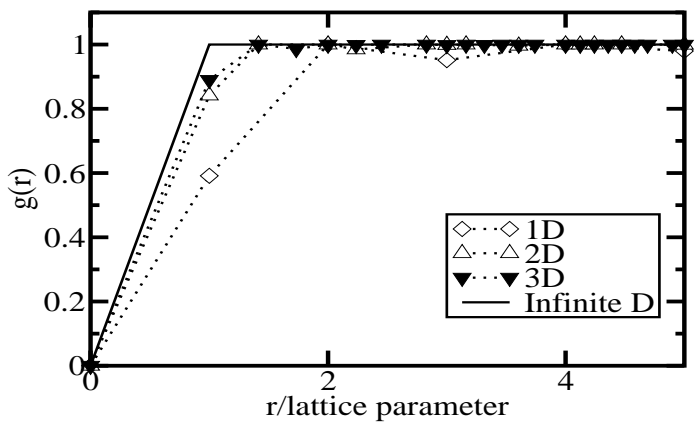

FIG. 1: Pair distribution functions of systems of noninteracting fermions on a lattice for different dimensions at half-filling. The Gutzwiller approximation uses the pair distribution function of the inifinite limit for all dimensions.

resentation. The hopping term, being the expectation value of an operator which is not diagonal in occupation number representation is approximated in a similar fashion as in the original GA. Implementation consists of Monte Carlo sampling first used to calculate the exact GWF by Yokoyama and Shiba. 40] It may also be possible to approximate the distribution with the exchange hole as was done for continuous systems of interacting fermions. [41, 42, 43] For lattices a two-site approximation which takes into account the exchange hole has been proposed by Razafimandimby [44].

To motivate the central idea of our approach the dimensional dependence of the exchange hole at half-filling is presented in Fig. 1. Due to exchange there is an effective repulsion between nearest-neighboring $(r=1)$ particles which is strongest in the case of one dimension, and it decreases with the number of dimensions (see also Ref. [44]). Further away from the origin $(r>1)$ the functions exhibit gradually decreasing oscillations.

Via comparison with the full GWF we show that such a procedure brings the GA into excellent agreement with the full GWF. The interaction energy is exact as a function of the variational parameter, and the hopping energy also shows close correspondence where full GWF results are available. The anti-ferromagnetic order parameter is also in excellent agreement between our approach and the full GWF calculation, while the original GA significantly underestimates antiferromagnetism.

\section{METHOD}

\section{A. The Hubbard Hamiltonian and the Gutzwiller wavefunction}

The Hubbard Hamiltonian [1, 2, 3, 4] is given by

$$
H=-t \sum_{\langle i, j\rangle \sigma}^{N} c_{i \sigma}^{\dagger} c_{j \sigma}+U \sum_{i=1}^{N} n_{i \uparrow} n_{i \downarrow}
$$

We will assume a system with $L$ lattice sites and with $N_{\uparrow}$ and $N_{\downarrow}$ particles with spins up and down respectively. For future reference we also define the anti-ferromagnetic order parameter as

$$
\left\langle M_{z}^{2}\right\rangle=\left\langle\left(\frac{1}{N} \sum_{i=1}^{N} \epsilon_{i} S_{z}(i)\right)^{2}\right\rangle,
$$

where $S_{z}(i)$ denotes the $z$-component of the spin at site $i, \epsilon_{i}$ is either 1 or -1 depending on which sublattice site $i$ belongs to.

The variational wavefunction to the Hubbard model with which we are concerned is the Gutzwiller wavefunction $(\mathrm{GWF})$

$$
|\Psi\rangle=\exp \left(-\gamma \sum_{i} n_{i \uparrow} n_{i \downarrow}\right)|F S\rangle,
$$

where $|F S\rangle$ indicates a Fermi sea of non-interacting fermions, the sum in the exponential counts the number of doubly occupied sites, and $\gamma$ is a variational parameter. For a homogeneous system the Fermi sea is formed by filling in the plane wave states with the lowest hopping energies,

$$
|F S\rangle=c_{\mathbf{k}_{\mathbf{1}} \uparrow}^{\dagger} \ldots c_{\mathbf{k}_{\mathbf{N}_{\uparrow} \uparrow}^{\dagger}}^{\dagger} c_{\mathbf{l}_{\mathbf{1}} \downarrow}^{\dagger} \ldots c_{\mathbf{l}_{\mathbf{N}_{\downarrow} \downarrow}^{\dagger}}|0\rangle .
$$

Eq. (4) can be rewritten in terms of sums over configurations in real-space as [7]

$$
\begin{aligned}
& |F S\rangle=L^{-\left(\frac{\left(N_{\uparrow}+N_{\downarrow}\right)}{2}\right)} \sum_{\{\mathbf{g}\}} \sum_{\{\mathbf{h}\}}\left|\begin{array}{ccc}
e^{i \mathbf{k}_{\mathbf{1}} \cdot \mathbf{g}_{\mathbf{1}}} & \cdots & e^{i \mathbf{k}_{\mathbf{1}} \cdot \mathbf{g}_{\mathbf{N}_{\uparrow}}} \\
\vdots & \ddots & \vdots \\
e^{i \mathbf{k}_{\mathbf{N}_{\uparrow}} \cdot \mathbf{g}_{1}} & \cdots & e^{i \mathbf{k}_{\mathbf{N}_{\uparrow}} \cdot \mathbf{g}_{\mathbf{N}_{\uparrow}}}
\end{array}\right|(5) \\
& \times\left|\begin{array}{ccc}
e^{i \mathbf{l}_{\mathbf{1}} \cdot \mathbf{h}_{\mathbf{1}}} & \cdots & e^{i \mathbf{1}_{\mathbf{1}} \cdot \mathbf{h}_{\mathbf{N}_{\downarrow}}} \\
\vdots & \ddots & \vdots \\
e^{i \mathbf{l}_{\mathbf{N}_{\downarrow}} \cdot \mathbf{h}_{\mathbf{1}}} & \cdots & e^{i \mathbf{l}_{\mathbf{N}_{\downarrow}} \cdot \mathbf{h}_{\mathbf{N}_{\downarrow}}}
\end{array}\right| c_{\mathbf{g}_{1} \uparrow}^{\dagger} \ldots c_{\mathbf{g}_{\mathbf{N}_{\uparrow} \uparrow}^{\dagger}} c_{\mathbf{h}_{1} \downarrow}^{\dagger} \ldots c_{\mathbf{h}_{\mathbf{N}_{\downarrow} \downarrow}^{\dagger}}^{\dagger}|0\rangle,
\end{aligned}
$$

where $\mathbf{g}$ and $\mathbf{h}$ denote the configurations of particles with spin up and down respectively. These configurations are such that at most only one particle of each spin can occupy a particular site. To save space we introduce the notation $\mathfrak{D}[\mathbf{k} ; \mathbf{g}]$ for the determinants. The normalization of the GWF can be written

$$
\begin{aligned}
\langle\Psi \mid \Psi\rangle= & L^{-\left(N_{\uparrow}+N_{\downarrow}\right)} \sum_{\{\mathbf{g}\}} \sum_{\{\mathbf{h}\}}|\mathfrak{D}[\mathbf{k} ; \mathbf{g}]|^{2} \\
& \times|\mathfrak{D}[\mathbf{l} ; \mathbf{h}]|^{2} \exp [-2 \gamma D(\mathbf{g}, \mathbf{h})],
\end{aligned}
$$


where $D(\mathbf{g}, \mathbf{h})$ denotes the number of double occupations for the particular configuration of up-spin and down-spin particles $\mathbf{g}$ and $\mathbf{h}$ respectively. One can define the probability distribution

$$
P_{G W F}(\mathbf{g}, \mathbf{h})=|\mathfrak{D}[\mathbf{k} ; \mathbf{g}]|^{2}|\mathfrak{D}[\mathbf{l} ; \mathbf{h}]|^{2} \exp [-2 \gamma D(\mathbf{g}, \mathbf{h})] .
$$

The exchange hole is obtained via tracing out all but two variables corresponding to particles with parallel spin in the distribution $P_{G W F}$ with $\gamma=0$. With the help of $P_{G W F}$ one can write expectation values diagonal in occupation representation. For example, the expectation value of the number of double occupations can be written as

$$
\left\langle\sum_{i} n_{i \uparrow} n_{i \downarrow}\right\rangle=\frac{\sum_{\{\mathbf{g}, \mathbf{h}\}} P_{G W F}(\mathbf{g}, \mathbf{h}) D(\mathbf{g}, \mathbf{h})}{\sum_{\{\mathbf{g}, \mathbf{h}\}} P_{G W F}(\mathbf{g}, \mathbf{h})} .
$$

The hopping term is not diagonal in the coordinate representation. For example, if a hopping of an up-spin particle between particular sites $i$ and $j$ is considered we have

$$
\begin{gathered}
\left\langle\Psi\left|c_{i \uparrow}^{\dagger} c_{j \uparrow}\right| \Psi\right\rangle=L^{-\left(N_{\uparrow}+N_{\downarrow}\right)} \sum_{\{\mathbf{g}\}}{ }^{\prime} \sum_{\{\mathbf{h}\}} \mathfrak{D}^{*}\left[\mathbf{k} ; \mathbf{g}^{\prime}{ }_{i j \uparrow}\right] \times \\
\mathfrak{D}[\mathbf{k} ; \mathbf{g}]|\mathfrak{D}[\mathbf{l} ; \mathbf{h}]|^{2} \exp \left[-\gamma\left\{D\left(\mathbf{g}^{\prime}{ }_{i j \uparrow}, \mathbf{h}\right)+D(\mathbf{g}, \mathbf{h})\right\}\right] .
\end{gathered}
$$

The hopping changes the configuration from $g$ to $g^{\prime}{ }_{i j \uparrow}$, hence the determinant as well as the projection term is in general altered. In Eq. (9) $\mathbf{g}_{i j \uparrow}^{\prime}$ denotes configurations with site $i$ occupied and site $j$ unoccupied and $\mathbf{g}$ configurations with site $i$ unoccupied and site $j$ occupied. The prime on the sum over the up-spin configurations indicates that only these types of configurations enter the summation, that allow for the hopping.

The hopping can leave the number of double occupations unchanged, increase or decrease it by one. Hence we can rewrite Eq. (9) as

$$
\left\langle\Psi\left|c_{i \uparrow}^{\dagger} c_{j \uparrow}\right| \Psi\right\rangle=L^{-\left(N_{\uparrow}+N_{\downarrow}\right)} \sum_{\{\mathbf{g}\}}{ }_{\{\mathbf{h}\}} \mathfrak{D}^{*}\left[\mathbf{k} ; \mathbf{g}^{\prime}{ }_{i j \uparrow}\right] \times(10)
$$

$\mathfrak{D}[\mathbf{k} ; \mathbf{g}]|\mathfrak{D}[\mathbf{l} ; \mathbf{h}]|^{2} \exp \left[-\gamma\left\{2 D(\mathbf{g}, \mathbf{h})+\Delta D\left(\mathbf{g}_{i j \uparrow}^{\prime}, \mathbf{g} ; \mathbf{h}\right)\right\}\right]$,

where $\Delta D\left(\mathbf{g}_{i j \uparrow}^{\prime}, \mathbf{g} ; \mathbf{h}\right)$ denotes the change in double occupation when an up-spin particle hops from site $j$ to $i$ (in other words the configuration changes from $\mathbf{g}$ to $\mathbf{g}_{i j \uparrow}^{\prime}$ ). We now define the estimators for hopping from site $j$ to $i$ as

$$
\begin{array}{r}
\chi_{G W F}^{i j \uparrow}(\mathbf{g}, \mathbf{h})=-t \mathfrak{D}^{*}\left[\mathbf{k} ; \mathbf{g}^{\prime}{ }_{i j \uparrow}\right] / \mathfrak{D}^{*}[\mathbf{k} ; \mathbf{g}] \times \\
\exp \left[-\gamma \Delta D\left(\mathbf{g}^{\prime}{ }_{i j \uparrow}, \mathbf{g} ; \mathbf{h}\right)\right], \\
\chi_{G W F}^{i j \downarrow}(\mathbf{g}, \mathbf{h})=-t \mathfrak{D}^{*}\left[\mathbf{l} ; \mathbf{h}^{\prime}{ }_{i j \downarrow}\right] / \mathfrak{D}^{*}[\mathbf{l} ; \mathbf{h}] \times \\
\exp \left[-\gamma \Delta D\left(\mathbf{g} ; \mathbf{h}^{\prime}{ }_{i j \uparrow}, \mathbf{h}\right)\right] .
\end{array}
$$

and use these definitions to write

$$
\tau_{G W F}^{i j \sigma}(\mathbf{g}, \mathbf{h})= \begin{cases}\chi_{G W F}^{i j \sigma}(\mathbf{g}, \mathbf{h}) & \text { if site } j \text { has a particle } \\ & \text { with spin } \sigma \text { and } \\ & \text { site } i \text { does not. } \\ 0 & \text { otherwise. }\end{cases}
$$

Eqs. (11) and (12) allow us to write the concatenated form for the estimator for the kinetic energy as

$$
T_{G W F}(\mathbf{g}, \mathbf{h})=\sum_{i j \sigma} \tau_{G W F}^{i j \sigma}(\mathbf{g}, \mathbf{h}),
$$

and write the expectation value of the total energy as

$$
E_{G W F}=\frac{\sum_{\{\mathbf{g}, \mathbf{h}\}} P_{G W F}(\mathbf{g}, \mathbf{h})\left\{T_{G W F}(\mathbf{g}, \mathbf{h})+U D(\mathbf{g}, \mathbf{h})\right\}}{\sum_{\{\mathbf{g}, \mathbf{h}\}} P_{G W F}(\mathbf{g}, \mathbf{h})}
$$

where the summations are now unrestricted. A Monte Carlo procedure can be constructed [40] to sample the distributions $P_{G W F}$ and evaluate the expectation values defined in (Eq. (14)).

The expectation value of the energy for the GWF was solved exactly in one dimension by Metzner and Vollhardt [9]. The Fermi step in this case is finite for all finite values of the interaction strength, hence the Gutzwiller wavefunction was shown to be metallic in one dimension. Millis and Coppersmith have later generalized this conclusion to any system of finite dimensions [13].

\section{B. The Gutzwiller approximation}

In the Gutzwiller approximation the determinant factors of the probability distribution $P_{G W F}$ and of the estimator $T_{G W F}$ (Eq. (10)) is replaced by configurational averages obtained from the non-interacting system. Our description of how this is done is based on Reference [7].

Considering only the up-spin channel one can write the normalization of the Fermi sea as

$$
\uparrow\langle F S \mid F S\rangle_{\uparrow}=L^{-N_{\uparrow}} \sum_{\mathbf{g}}|\mathfrak{D}[\mathbf{k} ; \mathbf{g}]|^{2}=1,
$$

since the wavefunctions that enter are normalized planewaves themselves. As the sum in Eq. (15) is over all configurations of up-spin particles on the lattice, such that at most one particle occupies a particular site we can approximate each term by its average as

$$
|\mathfrak{D}[\mathbf{k} ; \mathbf{g}]|^{2} \approx\left\langle|\mathfrak{D}[\mathbf{k} ; \mathbf{g}]|^{2}\right\rangle=\frac{L^{N_{\uparrow}}}{C_{N_{\uparrow}}^{L}},
$$

where $C_{N_{\uparrow}}^{L}$ denotes the number of ways $N_{\uparrow}$ particles can be placed on $L$ lattice sites. The down-spin particles can be handled similarly. This approximation results in a simplified probability distribution as compared to $P_{G W F}$

$$
P_{G A}(\mathbf{g}, \mathbf{h})=\exp [-2 \gamma D(\mathbf{g}, \mathbf{h})],
$$

allowing the rewriting of averages for quantities diagonal in the occupation number representation. For example the average number of double occupations in the GA can be written as

$$
\left\langle\sum_{i} n_{i \uparrow} n_{i \downarrow}\right\rangle=\frac{\sum_{\{\mathbf{g}, \mathbf{h}\}} P_{G A}(\mathbf{g}, \mathbf{h}) D(\mathbf{g}, \mathbf{h})}{\sum_{\{\mathbf{g}, \mathbf{h}\}} P_{G A}(\mathbf{g}, \mathbf{h})} .
$$


Approximating the kinetic energy is complicated by the fact that the hopping is not diagonal in the occupation number representation. Here a configurational average is needed for products of two determinants $\mathfrak{D}^{*}\left[\mathbf{k} ; \mathbf{g}^{\prime}\right] \mathfrak{D}[\mathbf{k} ; \mathbf{g}]$ for particular hopping terms. Considering only the up-spin channel one can write

$$
\begin{aligned}
\mathfrak{T} & ={ }_{\uparrow}\left\langle F S\left|c_{i \uparrow}^{\dagger} c_{j \uparrow}\right| F S\right\rangle_{\uparrow} \\
& =L^{-N_{\uparrow}} \sum_{\mathbf{g}} \mathfrak{D}^{*}\left[\mathbf{k} ; \mathbf{g}^{\prime}{ }_{i j \uparrow}\right] \mathfrak{D}[\mathbf{k} ; \mathbf{g}]
\end{aligned}
$$

One can also evaluate the average hopping over the Fermi sea explicitly as,

$$
\left.\mathfrak{T}=\frac{1}{L} \sum_{\mathbf{k}}^{*} \exp \left[i \mathbf{k} \cdot\left(\mathbf{R}_{\mathbf{i}}-\mathbf{R}_{\mathbf{j}}\right)\right)\right]
$$

where $\mathbf{R}_{\mathbf{i}}$ and $\mathbf{R}_{\mathbf{j}}$ denote the pair of lattice sites involved in the hopping, and the asterisk indicates that the sum be performed over occupied states only. The configurations entering the sum in Eq. (19) are the ones with one up-spin electron on site $j$ and site $i$ unoccupied. Of such configurations there are $C_{N_{\uparrow}-1}^{L-2}$. Hence the approximation

$$
\mathfrak{D}^{*}\left[\mathbf{k} ; \mathbf{g}^{\prime}{ }_{i j \uparrow}\right] \mathfrak{D}[\mathbf{k} ; \mathbf{g}] \approx\left\langle\mathfrak{D}^{*}\left[\mathbf{k} ; \mathbf{g}^{\prime}{ }_{i j \uparrow}\right] \mathfrak{D}[\mathbf{k} ; \mathbf{g}]\right\rangle=\mathfrak{T} \frac{L^{N_{\uparrow}}}{C_{N_{\uparrow}-1}^{L-2}}
$$

can be introduced. Using this approximation the average hopping of an up-spin particle from site $j$ to site $i$ can be written

$$
\begin{aligned}
& \frac{\left\langle\Psi\left|c_{i \uparrow}^{\dagger} c_{j \uparrow}\right| \Psi\right\rangle}{\langle\Psi \mid \Psi\rangle}=\mathfrak{T} \frac{C_{N_{\uparrow}}^{L}}{C_{N_{\uparrow}-1}^{L-2}} \times \\
& \frac{\sum_{\{\mathbf{g}\}}^{\prime} \sum_{\{\mathbf{h}\}} P_{G A}(\mathbf{g}, \mathbf{h}) \exp \left[-\gamma \Delta D\left(\mathbf{g}^{\prime}{ }_{i j \uparrow}, \mathbf{g} ; \mathbf{h}\right)\right]}{\sum_{\{\mathbf{g}\}} \sum_{\{\mathbf{h}\}} P_{G A}(\mathbf{g}, \mathbf{h})}
\end{aligned}
$$

Eq. (22) is an approximate expression for the hopping energy. It allows to formulate an estimator for the hopping which is diagonal in the occupation number representation. We can then write the estimator for the hopping from site $j$ to site $i$ (the effective operator which is averaged in order to calculate the hopping energy) as

$$
\begin{aligned}
& \chi_{G A}^{i j \uparrow}(\mathbf{g} ; \mathbf{h})=\tilde{\mathfrak{T}}_{\uparrow} \exp \left[-\gamma \Delta D\left(\mathbf{g}_{i j \uparrow}^{\prime}, \mathbf{g} ; \mathbf{h}\right)\right], \\
& \chi_{G A}^{i j \downarrow}(\mathbf{g} ; \mathbf{h})=\tilde{\mathfrak{T}}_{\downarrow} \exp \left[-\gamma \Delta D\left(\mathbf{g} ; \mathbf{h}^{\prime}{ }_{i j \downarrow}, \mathbf{h}\right)\right],
\end{aligned}
$$

where

$$
\tilde{\mathfrak{T}}_{\sigma}=\mathfrak{T} \frac{C_{N_{\sigma}}^{L}}{C_{N_{\sigma}-1}^{L-2}}
$$

We can use these definitions to write

$$
\tau_{G A}^{i j \sigma}(\mathbf{g}, \mathbf{h})= \begin{cases}\chi_{G A}^{i j \sigma}(\mathbf{g}, \mathbf{h}) & \text { if site } j \text { has a particle } \\ & \text { with spin } \sigma \text { and } \\ & \text { site } i \text { does not. } \\ 0 & \text { otherwise. }\end{cases}
$$

Eqs. (23) and (25) allow us to write the concatenated form for estimator of the kinetic energy again as an unrestricted sum

$$
T_{G A}(\mathbf{g}, \mathbf{h})=\sum_{i j \sigma} \tau_{G A}^{i j \sigma}(\mathbf{g}, \mathbf{h}),
$$

resulting in the expectation value of the total energy as

$$
E_{G A}=\frac{\sum_{\{\mathbf{g}, \mathbf{h}\}} P_{G A}(\mathbf{g}, \mathbf{h})\left\{T_{G A}(\mathbf{g}, \mathbf{h})+U D(\mathbf{g}, \mathbf{h})\right\}}{\sum_{\{\mathbf{g}, \mathbf{h}\}} P_{G A}(\mathbf{g}, \mathbf{h})} .
$$

Note that for the non-interacting system $(U=0)$ the energy is exact by construction (one could also define the constants $\tilde{\mathfrak{T}}_{\sigma}$ from this condition). In summary the estimator for the hopping is the product of a scaling factor and a factor which accounts for the change in the number of double occupations caused by the hopping itself.

In summary the GA can be considered a two-step approximation: the exact distribution $P_{G W F}$, which is complicated by the determinant factors, is replaced by the simpler $P_{G A}$, and the estimator for the hopping $T_{G W F}$ is replaced by $T_{G A}$. In the $T_{G W F}$ the quotient of determinants, which originates from the fact that the hopping is not a diagonal operator in the occupation number representation is replaced by an average value to arrive at the approximation $T_{G A}$. The approximate estimator $T_{G A}$ is diagonal in the occupation number representation.

In the case of the GA the average energy (Eq. (27)) and other relevant observables can be evaluated analytically [3, 4, 7, 8, 19]. The resulting MIT, known as the Brinkman-Rice transition [8], is characterized by the vanishing of the expectation value of the double occupations as well as that of the hopping energy. The latter can be shown to be a result of the closure of the Fermi step [7, 8, 19].

\section{Implementing the exchange hole}

In the following we investigate the effect of the exchange hole on the Gutzwiller approximation. To this end we substitute $P_{G W F}$ for $P_{G A}$ in Eq. (27). We also scale the constants $\mathfrak{T}_{\sigma}$ so that the hopping energy remains exact in the non-interacting limit. We refer to this approximation scheme as the GA-X. For the expression of the energy we can write

$E_{G A-X}=\frac{\sum_{\{\mathbf{g}, \mathbf{h}\}} P_{G W F}(\mathbf{g}, \mathbf{h})\left\{T_{G A-X}(\mathbf{g}, \mathbf{h})+U D(\mathbf{g}, \mathbf{h})\right\}}{\sum_{\{\mathbf{g}, \mathbf{h}\}} P_{G W F}(\mathbf{g}, \mathbf{h})}$.

The estimator for the hopping $T_{G A-X}$ has the same form as $T_{G A}$, the only difference is the scaling factor to satisfy the condition in the non-interacting limit.

The expression for the average number of double occupations as a function of the variational parameter $\gamma$ in our scheme is the same as in the exact GWF case (Eq. (8)). This does not necessarily mean that we obtain the 
exact expectation value of this operator, however. Since the hopping is approximated as a function of the parameters $t$ and $U$, the minimization in the parameter $\gamma$ does not guarantee that the exact $\gamma$ is obtained.

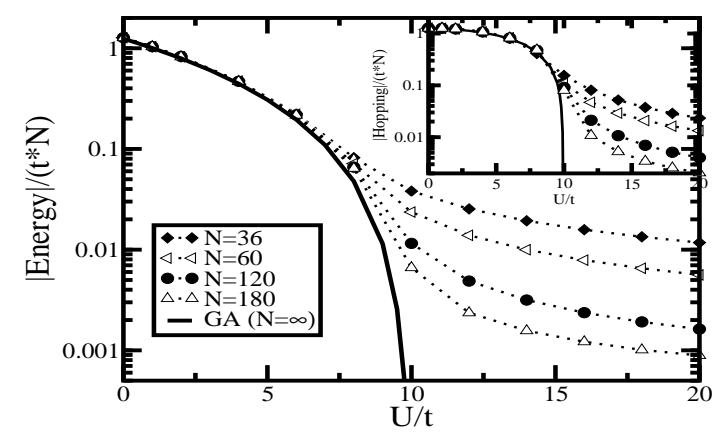

FIG. 2: Absolute value of the total energy per particle calculated in the Gutzwiller approximation: Monte Carlo results for various system sizes and analytical results for the thermodynamic limit. The inset shows the absolute value of the hopping energy.

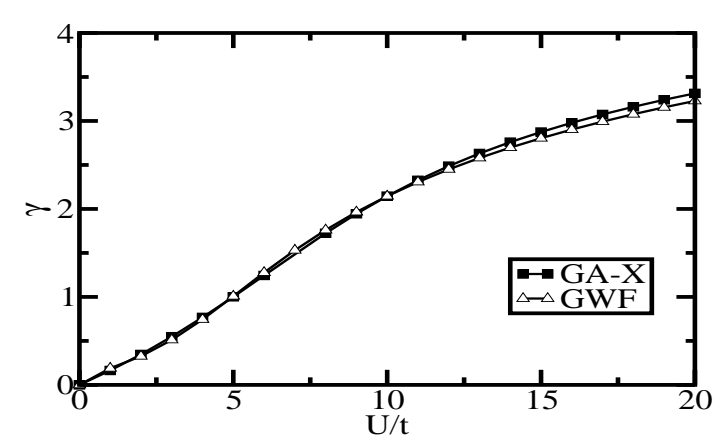

FIG. 3: Variational parameter $\gamma$ as a function of the interaction parameter $U$.

\section{Monte Carlo sampling}

To sample the distributions described in the previous subsection we apply the Monte Carlo method on a lattice due to Yokoyama and Shiba [40]. Our system consists of a fixed number of sites $L$ and up-spin and down-spin particles $N_{\uparrow}$ and $N_{\downarrow}$ respectively. Our Monte Carlo method consists of generating configurations with at most one particle of each spin on each lattice site. We generate

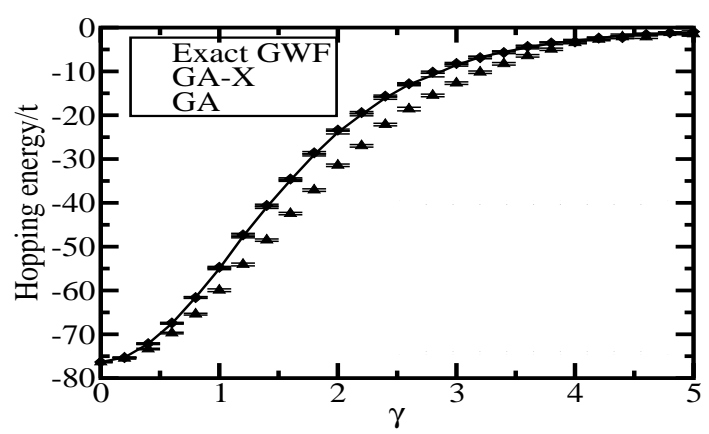

FIG. 4: Hopping energy calculated via the Monte Carlo sampling [40] of the Gutzwiller wavefunction, the Gutzwiller approximation with the exchange hole, and the standard Gutzwiller approximation for a system of 60 sites as a function of the variational parameter $\gamma$.

the configurations using two types of moves. We attempt moves of particles of a particular spin to sites without particles of that spin. We also attempt exchange moves between sites occupied by particles of opposite spin, and between sites which are doubly occupied and empty. We checked our Monte Carlo code against a full GWF calculation based on exact diagonalization for 12 sites and found excellent agreement.

\section{RESULTS}

In the following we apply the method described above to the one-dimensional Hubbard model at half-filling. In order to test our Monte Carlo program we perform calculations for GA. We have performed calculations with sizes up to 180 . The result in the thermodynamic limit is also known [7]. All of these calculations are based on MC runs of on the order of $10^{6}$ steps. The hopping and interaction energies were calculated for an $U=1$ system. In order to stabilize the search for the minimum, for all of the following calculations, we fitted the calculated data points [45]. The results for the absolute value of the total energy are shown in Fig. 2 as well as the absolute value of the kinetic energy shown in the inset. The Brinkman-Rice transition is clearly visible for the curve corresponding to the thermodynamic limit. The calculations of systems with different sizes converge to the curve in the thermodynamic limit, in particular they converge to zero in the region of $U$ where the Brinkman-Rice transition predicts insulating behavior (where the energy is zero).

We note that often the Fermi step is taken as the indicator of the Brinkman-Rice MIT [7, 40]. To calculate the Fermi step the density of states in momentum space is 


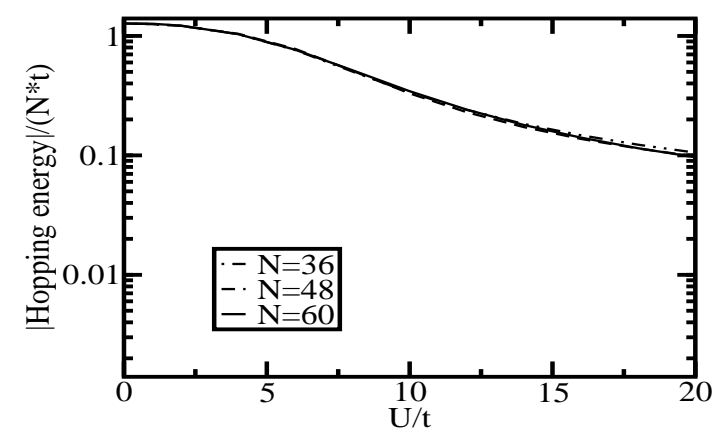

FIG. 5: Absolute value of the hopping energy calculated from the GA-X approximation for three different system sizes.

calculated. One can argue that as the Fermi step closes the hopping energy becomes zero, and for an open Fermi step the hopping energy has to be finite [7]. Hence we can take the hopping energy as an indicator of the MIT. Moreover, the interaction energy, or rather the number of double occupations, is zero as well.

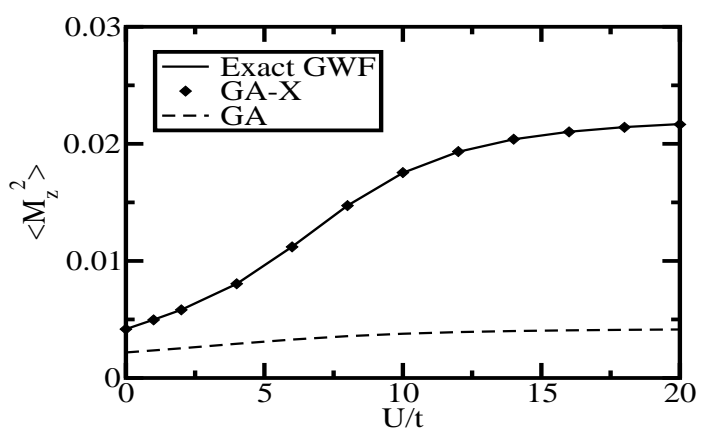

FIG. 6: Comparison of the antiferromagnetic order parameter (defined in Eq. (2)) for the exact Gutzwiller wavefunction, GA-X, and GA.

In Fig. 3 the variational parameter $\gamma$ is shown as a function of the interaction parameter $U$ for the full GWF and the GA-X calculations. Quantitative agreement is found between the two curves. In Fig. 4 the hopping energies as a function of the variational parameter $\gamma$ are compared from an exact calculation for the full GWF, the GA-X, and the GA schemes for a system at half-filling with 60 lattice sites. In the case of 60 sites Monte Carlo sampling [40] was used in all cases. The agreement between the exact GWF and GA-X is excellent, the exact curve essentially coincides with the GA-X results. GWF and GA-X differ only in the definition of the estimator for the kinetic energy. The fact that the kinetic energies of the two approaches essentially coincide is indirect evidence that the GA-X approximation is metallic. More evidence for this conclusion is provided by comparing the hopping energy for different system sizes, shown in Fig. 5 for sizes 36, 48, and 60. The hopping energy, whose becoming zero indicates the Brinkman-Rice MIT, shows negligible size-independence and does not become zero in the range of $U$ considered (whereas the size-dependence is strong for the GA (Fig. 20).

Since the interaction energy as a function of the variational parameter is identical for the GWF and GA-X, our numerical evidence suggests that the GWF and the GA-X coincide. It is of interest to note that the GA and the GWF coincide in the case of infinite dimensions, where the form of the exchange hole assumed in the GA coincide with the exact exchange hole. Our results indicate that implementing the exact exchange hole in one dimension brings the GA into agreement with the GWF in one dimension.

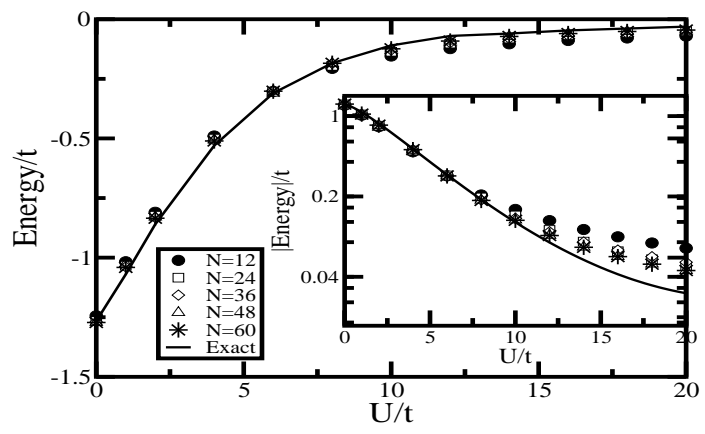

FIG. 7: Absolute value of the total energy per particle calculated via the GA-X: Monte Carlo results for various system sizes and the results for the thermodynamic limit of the full GWF (Ref. [9, 10]). The inset (logarithmic in energy) shows that the finite size dependence of the energy increases with the interaction strength $U$.

In Fig. 6] we present the anti-ferromagnetic order parameter (defined in Eq. (21)) for a system of 60 lattice sites. The three different methods GWF, GA-X, and GA are compared. Implementation of the exchange hole recovers anti-ferromagnetism entirely, a results that can be anticipated from the results on the hopping energy (Fig. (4). We stress that metallicity and anti-ferromagnetism are recovered together as the exchange hole is implemented. In Refs. [46, 47] expressions are given for the spin-correlation functions. Using these expressions we have calculated the antiferromagnetic order parameter defined in Eq. (2) and we have obtained a value of $\left\langle M_{z}^{2}\right\rangle=0.0201$ for a system of 60 sites with $\gamma \rightarrow \infty$ in 


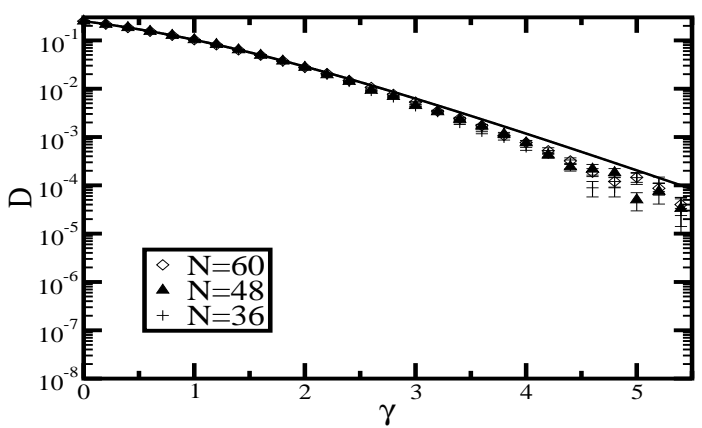

FIG. 8: Double occupation (shown on a logarithmic scale) calculated via GA-X and the thermodynamic limit (Ref. 9, 10]).

good agreement with our result of $\left\langle M_{z}^{2}\right\rangle=0.022 \pm 0.005$. Our recovery of metallic behaviour is related to the recovery of anti-ferromagnetic behavior.

In Figs. 7 and 8 we present a comparison of the total energies and the double occupations between various system sizes. In the inset in Fig. 7, which shows the absolute value of the energy on a logarithmic scale, the energies appear to converge to the exact solution of the GWF in the thermodynamic limit. The logarithmic deviations appear to be larger at large $U$. The average number of double occupations as a function of the variational parameter $\gamma$ are shown in Fig. 8. The exact expression for the number of double occupations in the thermodynamic limit as a function of $\gamma$ for the GWF wavefunction is given in Refs. [9, 10]. The agreement between the exact result and the GA-X results is goo for lower values of $\gamma$, discrepancies appear only at large values of $\gamma$, noticible mainly at values larger than the value that minimizes the energy at $U=20$ (see Fig. (3)). The discrepancy can be partly attributed to finite size effects, which tend to oversetimate ordering, and thereby suppress double occupations, and also the difficulty in sampling with the standard MC method [40] in systems which are approaching a critical point $(\gamma \rightarrow \infty)$.

\section{CONCLUSIONS}

We have investigated the effect of implementing the exchange hole in the Gutzwiller approximation in one dimension for the Hubbard model at half-filling. The estimator used for the hopping energy was taken from the Gutzwiller approximate solution, but the distribution of configurations was the one corresponding to the exact solution of the Gutzwiller wavefunction, with the exchange hole implemented. Comparison with exact calculations based on the Gutzwiller wavefunction were presented.

The resulting approximation is in excellent quantitative agreement with the exact result. The approximate hopping and the hopping of the exact solution of the GWF are in excellent agreement. When compared to the Gutzwiller approximation we find that through implementing the exchange hole metallicity and antiferromagnetism of the full GWF are both recovered. Our essential conclusion here is that by implementing the exchange hole one can account for the anti-ferromagnetic correlations present in the exact GWF, and this procedure results in the recovery of metallicity.

As is well known 11, 12, 21 the Gutzwiller approximation is exact in the limit of infinite dimensionality. What our results suggest is that implementation of the exchange hole brings the Gutzwiller approximation into agreement with the full GWF results. In the future we plan to study this question in more than one finite dimensions.

From a methodological point of view our result may lead to improvements in the future. It is possible to construct approximate potentials for the exchange hole [41, 42, 43]. Such an approximate potential could then be sampled which would result in an approximate GWF, or the exact distribution could be sampled facilitated by the approximated potential via umbrella sampling [48], stochastic potential switching [49] or accelerated Monte Carlo methods [50, 51, 52, 53].

\section{ACKNOWLEDGMENTS}

$\mathrm{BH}$ is supported by FWF (Fondsförderung der wissenschaftlichen Forschung) grant number P21240-N16. Beneficial discussions with L. Chioncel and E. Arrigoni are gratefully acknowledged.
[1] J. Hubbard, Proc. Roy. Soc. London Ser. A, A276 238 (1963).

[2] J. Kanamori, Prog. Theoret. Phys., 30275 (1963).

[3] M. C. Gutzwiller, Phys. Rev. Lett., 10159 (1963).

[4] M. C. Gutzwiller, Phys. Rev., 137 A1726 (1965).

[5] M. Imada, A Fujimori, and Y. Tokura, Rev. Mod. Phys., 701039 (1998).

[6] A. Auerbach, Interacting Electrons and Quantum Magnetism, Springer (1998).

[7] P. Fazekas, Lecture Notes on Electron Correlation and
Magnetism, World Scientific (1999).

[8] W. F. Brinkman and T. M. Rice, Phys. Rev. B, 24302 (1970).

[9] W. Metzner and D. Vollhardt, Phys. Rev. Lett., 59121 (1987).

[10] W. Metzner and D. Vollhardt, Phys. Rev. B, 377382 (1988).

[11] W. Metzner and D. Vollhardt, Phys. Rev. Lett., 62324 (1989).

[12] W. Metzner and D. Vollhardt, Helv. Phys. Acta, 63364 
(1990).

[13] A. J. Millis and S. N. Coppersmith, Phys. Rev. B, 43 13770 (1991).

[14] E. H. Lieb and F. Y. Wu, Phys. Rev. Lett., 201445 (1968).

[15] D. Baeriswyl, Found. Physics, 302033 (2000).

[16] M. Capello, F. Becca, M. Fabrizio, S. Sorella, and E. Tosatti, Phys. Rev. Lett., 94026406 (2005).

[17] D. Tahara and M. Imada, J. Phys. Soc. Jpn., 77093703 (2008).

[18] B. Edegger, V. N. Muthukumar, and C. Gros, Adv. Phys., 56927 (2007).

[19] D. Vollhardt, Rev. Mod. Phys., 5699 (1984).

[20] T. A. Kaplan, P. Horsch, and P. Fulde, Phys. Rev. Lett., 49889 (1982).

[21] G. Kotliar and A. E. Ruckenstein, Phys. Rev. Lett., 57 1362 (1986).

[22] W. Metzner, Z. Physik B Condens. Matter, 77253 (1989).

[23] P.G.J. van Dongen, F. Gebhard, and D. Vollhardt, Z. Physik B Condens. Matter, 76199 (1989).

[24] Z. Gulácsi, M. Gulácsi, and J. Boldizsár, Phys. Rev. B 474168 (1993).

[25] Z. Gulácsi and M. Gulácsi, Philos. Mag. B 69437 (1994).

[26] Z. Gulácsi and M. Gulácsi, Phys. Rev. B 441475 (1991).

[27] H. Yokoyama, Prog. Theor. Phys 10859 (2002).

[28] G. D. Mahan, Many-Particle Physics, 3rd Ed., Kluwer Academic, New York, (2000).

[29] G. Seibold and J. Lorenzana, Phys. Rev. Lett., 862605 (2001).

[30] J. Bünemann, F. Gebhard, and W. Weber, Found. Phys., 302011 (2000).

[31] J. Bünemann, D. Rasch, and F. Gebhard, J. Phys. Condens. Matter, 19436206 (2007).

[32] B. Edegger, N. Fukushima, C. Gros, and V. N. Muthukumar, Phys. Rev. B, 72134504 (2005).

[33] N. Fukushima, B. Edegger, V. N. Muthukumar, and C. Gros, Phys. Rev. B, 72144505 (2005).

[34] F. C. Zhang, C. Gros, T. M. Rice, and H. Shiba, Supercond. Sci. Tech., 136 (1988).

[35] B. Edegger, V. N. Muthukumar, C. Gros, and P. W. Anderson, Phys. Rev. Lett., 96207002 (2006).
[36] C. Gros, B. Edegger, V. N. Muthukumar, and P. W. Anderson, Proc. Nat. Acad. Sci. USA, 10314298 (2006).

[37] P. W. Anderson, Mat. Res. Bull., 8153 (1973).

[38] P. Fazekas and P. W. Anderson, Phil. Mag., 30432 (1974).

[39] P. W. Anderson, P. A. Lee, M. Randeria, T. M. Rice, N. Trivedi, and F. C. Zhang J. Phys. Condens. Matter, 16 R755 (2003).

[40] H. Yokoyama and H. Shiba, J. Phys. Soc. Japan, 561490 (1986).

[41] F. Lado, J. Chem. Phys., 475369 (1967).

[42] F. A. Stevens and M. A. Pokrant, Phys. Rev. A, 8990 (1967).

[43] B. Hetényi, L. Brualla, and S. Fantoni Phys. Rev. Lett., 93170202 (2004).

[44] H. A. Razafimandimby Z. Physik B - Condensed Matter, 4933 (1982).

[45] We used the following fitting procedure in determining continuous curves for the hopping, interaction energies, and the anti-ferromagnetic order parameter. We used the software GNUPLOT version 4.2. For each set of data (data points for one particular system size), we first optimized a function of the form $f(x)=A \exp \left(-B x^{C}\right)$, then reoptimized using the functional form $f(x)=$ $A \exp \left(-B x^{C}-D x^{E}\right)$. We found excellent agreement in all cases.

[46] F. Gebhard and D. Vollhardt Phys. Rev. Lett., 591472 (1987).

[47] F. Gebhard and D. Vollhardt Phys. Rev. B, 386911 (1987).

[48] G. M. Torrie and J. P. Valleau J. Comp. Phys., 23187 (1977).

[49] C. H. Mak J. Chem. Phys., 122214110 (2005).

[50] R. Iftimie, D. Salahub, D. Q. Wei, and J. Schofield J. Chem. Phys., 1134852 (2000).

[51] B. Hetényi, K. Bernacki, B. J. Berne J. Chem. Phys., 1178203 (2002).

[52] K. Bernacki, B. Hetényi, and B. J. Berne J. Chem. Phys., 12144 (2004).

[53] L. D. Gelb J. Chem. Phys., 1187747 (2003). 